\title{
Localization for Random Schrödinger Operators with Correlated Potentials
}

\author{
Henrique von Dreifus ${ }^{1 \star \star \star}$ and Abel Klein ${ }^{2 \star \star \star}$ \\ 1 Department of Physics, Princeton University, Princeton, NJ 08544, USA \\ 2 Department of Mathematics, University of California at Irvine, Irvine, CA 92717, USA
}

Received October 25, 1990; in revised form December 7, 1990

\begin{abstract}
We prove localization at high disorder or low energy for lattice Schrödinger operators with random potentials whose values at different lattice sites are correlated over large distances. The class of admissible random potentials for our multiscale analysis includes potentials with a stationary Gaussian distribution whose covariance function $C(x, y)$ decays as $|x-y|^{-\theta}$, where $\theta>0$ can be arbitrarily small, and potentials whose probability distribution is a completely analytical Gibbs measure. The result for Gaussian potentials depends on a multivariable form of Nelson's best possible hypercontractive estimate.
\end{abstract}

\section{Introduction}

We consider the random Schrödinger operator $H=-\Delta+V$ on $l^{2}\left(\mathbf{Z}^{d}\right)$, where $\Delta$ is the centered finite difference Laplacian, i.e., $\Delta(x, y)=1$ if $|x-y|=1$ and zero otherwise, and $V$ is an ergodic potential, i.e., $\left\{V(x) ; x \in \mathbf{Z}^{d}\right\}$ is an ergodic stochastic process. The motivation for studying this class of operators comes from Solid State Physics, where one is interested in the behavior of an electron in a random background. This model was first introduced by Anderson [1] and is known as the Anderson tight-binding model.

It is well known that the spectrum of the random operator $H$ is independent of the choice of potential with probability one $[2,3,4]$. The same is true of the decomposition of the spectrum into pure point, absolutely continuous and singular continuous spectrum $[3,4]$.

The random operator $H$ exhibits localization in an energy interval $I$ if it has only pure point spectrum in $I$ with probability one. In this case, if the eigenfunc-

\footnotetext{
* Current address: Instituto de Mathematica e Estatistica, Universidade de São Paulo, São Paulo, S.P., Brazil

$\star \star$ Partially supported by the NSF under grant PHY8515288

$\star \star \star$ Partially supported by the NSF under grant DMS8905627
} 
tions corresponding to eigenvalues in $I$ decay exponentially (polynomially), we say we have exponential (polynomial) localization.

Exponential localization for the Anderson tight-binding model is well understood in one dimension (e.g., [4]), where it was first established in the continuum by Gold'sheid, Molchanov and Pastur [5]. When the $\left\{V(x), x \in \mathbf{Z}^{d}\right\}$ are independent identically distributed random variables, one always has exponential localization $[3,6-9]$. The same is true on the strip [10,11]. For arbitrary ergodic potentials localization also holds if the joint conditional probability of the values of the potential at two neighboring sites with respect to the values of the potential in the remaining sites has an absolutely continuous component $[12,13,4]$. In particular, Simon [12] noticed that the result holds for nondeterministic ergodic Gaussian potentials and for potentials whose probability distribution is a Gibbs measure with an "a priori" measure with an absolutely continuous component and finite range interactions. The result for Gaussian potentials was also noticed by Pastur [14] (see also [4]). In one dimension exponential localization has also been proved for quasi-periodic potentials with large coupling constant [15-17].

In more than one dimension localization results had only been proven for independent potentials. For such potentials exponential localization was proven at either high disorder or low energy $[18,6,19,20,21,9]$.

In this article we prove localization at high disorder or low energy for certain correlated potentials, in any dimension. The class of admissible potentials include potentials whose values at different lattice sites are strongly correlated over large distances. Our proof yields only polynomial localization.

Our proof involves a multiscale analysis based on the ideas used by von Dreifus and Spencer [21, 22] and by von Dreifus and Klein [9] to give a simpler proof of localization in the independent case.

The class of potentials for which we prove localization includes stationary Gaussian potentials whose covariance function $C(x, y)$ decays as $|x-y|^{-\theta}$, where $\theta>0$ can be arbitrarily small, and potentials whose probability distribution is a completely analytical Gibbs measure. The result for Gaussian potentials depends on a multivariable form of Nelson's best possible hypercontractive estimate; for Gibbs potentials we use the work of Dobrushin and Shlosman [23, 24].

\section{Statement of Results}

We start with some notations and definitions. If $\Lambda \subset \mathbf{Z}^{d}$, we denote by $H_{\Lambda}$ the operator $H$ restricted to $l^{2}(\Lambda)$ with zero boundary condition outside $\Lambda$. The corresponding Green's function is $G_{\Lambda}(z)=\left(H_{\Lambda}-z\right)^{-1}$, defined for $z \notin \sigma\left(H_{\Lambda}\right)$. We will write $G_{\Lambda}(z ; x, y)=\left(H_{\Lambda}-z\right)^{-1}(x, y)$ for $x, y \in \Lambda$. If $\Lambda=\mathbf{Z}^{d}$, we simply write $G(z ; x, y)$. Notice that we omit the dependence of $H_{\Lambda}$ and $G_{\Lambda}$ on the potential $V$.

If $x \in \mathbf{Z}^{d}, x=\left(x_{1}, \ldots, x_{d}\right)$, we let $\|x\|=\|x\|_{\infty}=\max \left\{\left|x_{1}\right|, \ldots,\left|x_{d}\right|\right\}$. It will be convenient to use this norm; distances in $\mathbf{Z}^{d}$ will always be taken with respect to this norm. Occassionally we may also need the usual Euclidean norm $|x|=\|x\|_{2}=$ $\left(x_{1}^{2}+\cdots+x_{d}^{2}\right)^{1 / 2}$.

We will denote by $\mathbf{E}$ and $\mathbf{P}$ the expectation and probability measure on the underlying probability space for the stochastic process $\left\{V(x), x \in \mathbf{Z}^{d}\right\}$. 
Definition. The ergodic potential $V$ is a Wegner potential if the conditional probability distribution of $V(0)$ given $V(0)^{\perp}=\left\{V(x), x \in \mathbf{Z}^{d} \backslash\{0\}\right\}$ is absolutely continuous with respect to Lebesgue measure for $\mathbf{P}$-a.e. $V(0)^{\perp}$, and

$$
\delta \equiv\left\|\frac{\mathbf{P}\left(d V(0) \mid V(0)^{\perp}\right)}{d V(0)}\right\|_{\infty}<\infty,
$$

where the $L^{\infty}$-norm is taken with respect to $\mathbf{P}$.

If $V$ is a Wegner potential, Wegner's estimate on the density of states $[25,18,8,4]$ applies and we have

$$
\mathbf{P}\left\{d\left(E, \sigma\left(H_{\Lambda}\right)\right) \leqq \varepsilon\right\} \leqq \frac{2}{\delta} \varepsilon|\Lambda|
$$

for all $E \in \mathbf{R}, \varepsilon>0, \Lambda \subset \mathbf{Z}^{d}$. Furthermore, Kotani's trick can be used so the analysis of Simon and Wolff [20] and Delyon, Levi and Souillard [19] can be applied to Wegner potentials.

Let $x \in \mathbf{Z}^{d}, L>0$. We will denote by $\Lambda_{L}(x)$ the cube centered at $x$ with sides of length $L$, i.e.,

$$
\Lambda_{L}(x)=\left\{y \in \mathbf{Z}^{d} ;\|x-y\| \leqq \frac{L}{2}\right\} .
$$

Notice that $\left|\Lambda_{L}(x)\right| \leqq(L+1)^{d}$.

Given $\Lambda \subset \mathbf{Z}^{d}$ an event $A$ on $\Lambda$ is an event that depends only on $\{V(y) ; y \in \Lambda\}$. If $A$ is an event on the cube $\Lambda(0)$, we will use $A(x)$ to denote the same event shifted to the cube $\Lambda_{L}(x)$.

Definition. Let $1<\alpha<\rho, K \in\{2,3,4, \ldots\}$. We will say that the ergodic potential $V$ is of type $(\alpha, \rho, K)$ if for all $L$ sufficiently large, given $x_{1}, \ldots, x_{K} \in \mathbf{Z}^{d}$ with $\left\|x_{i}-x_{j}\right\| \geqq$ $\frac{1}{2} L^{\alpha}$ for $i \neq j$, and any event $A$ on $\Lambda_{L}(0)$, we have

$$
\mathbf{P}\left\{\bigcap_{i=1}^{\mathrm{K}} A\left(x_{i}\right)\right\} \leqq c \mathbf{P}\{A\}^{\rho}
$$

for some constant $c<\infty$.

If $V$ is an independent potential, i.e., the $V(x), x \in \mathbf{Z}^{d}$, are i.i.d.r.v.'s, then $V$ is of type $(\alpha, K, K)$ for any $1<\alpha<K, K=2,3, \ldots$. In this case we have equality in (2.2) with $c=1$.

Our main result is

Theorem 2.1. Let $V$ be a Wegner potential of type $(\alpha, \rho, K)$ for some $1<\alpha<\rho, K \in$ $\{2,3,4, \ldots\}$. Then the random Schrödinger operator $H=-\Delta+V$ exhibits polynomial localization at high disorder or low energy. More precisely; there exist $\eta_{0}>0$ such that:

(i) Let $H_{\lambda}=-\Delta+\lambda V, \lambda \in \mathbf{R}$. Then for $\eta>\eta_{0}$ we can find $\lambda(\eta)>0$ such that for any $\lambda$ with $|\lambda|>\lambda(\eta) H_{\lambda}$ has pure point spectrum with probability one and the corresponding eigenfunctions decay at least as fast as $\|x\|^{-\eta}$.

(ii) For any $\eta>\eta_{0}$ we can find $E(\eta)>0$ such that $H$ has pure point spectrum in $(-\infty,-E(\eta)) \cup(E(\eta), \infty)$ with probability one and the corresponding eigenfunctions decay at least as fast as $\|x\|^{-\eta}$. 
Theorem 2.1 will be proven by a multiscale analysis in Sect. 3. Condition (2.2) will replace independence in our proof, the price we pay is that the proof only yields polynomial localization. But condition (2.2) is satisfied by potentials that can be strongly correlated at large distances.

We give two examples of ergodic potentials satisfying the hypotheses of Theorem 2.1: Gaussian and Gibbs potentials.

We start with Gaussian potentials, which we can take to have mean zero without loss of generality.

Theorem 2.2. Let $V$ be a stationary Gaussian process with mean zero and covariance $C(x, y)=\mathbf{E}(V(x) V(y))$ for $x, y \in \mathbf{Z}^{d}$. Suppose:

(i) $C_{\Lambda}$ is invertible for all $\Lambda \subset \mathbf{Z}^{d}$ finite and

$$
a \equiv \sup \left\{C_{\Lambda}^{-1}(0,0) ; 0 \in \Lambda \subset \mathbf{Z}^{d} \text { finite }\right\}<\infty,
$$

(ii)

where $C_{\Lambda}$ is the matrix $\{C(x, y)\}_{x, y \in \Lambda}$.

$$
|C(x, y)| \leqq \frac{b}{1+\|x-y\|^{\theta}}
$$

for some $\theta>0, b<\infty$ and all $x, y \in \mathbf{Z}^{d}$.

Then there exists $\tau, 0 \leqq \tau \leqq d$, such that if $\max \left\{1, \frac{d+\tau}{\theta}\right\}<\alpha<\rho<2^{k}, k a$ positive integer, we have that $V$ is a Wegner potential of type $\left(\alpha, \rho, 2^{k}\right)$.

In particular, $H=-\Delta+V$ exhibits polynomial localization at high disorder or low energy, in the precise sense of Theorem 2.1.

Remarks. Ergodicity follows from (2.4). Notice that

$$
C_{\Lambda}^{-1}(0,0)=\frac{\operatorname{det} C_{\Lambda \backslash\{0\}}}{\operatorname{det} C_{\Lambda}}
$$

If $d=1,(2.3)$ is equivalent to the Gaussian process being nondeterministic (e.g., $[26,27])$. If the operator $C$ on $l^{2}\left(\mathbf{Z}^{d}\right)$ with kernel given by the covariance function $C(x, y)$ is strictly positive, i.e., $C \geqq w I>0$, then $(2.3)$ holds with $a \leqq 1 / w$. In this case we will see that we can take $\tau=0$. Notice that the exponent $\theta$ in (2.4) can be arbitrarily small so the values of the Gaussian potential can be strongly correlated at large distances.

Examples of such Gaussian potentials can be given by specifying the covariance operator $C$. For instance let $\tilde{\Delta}=\Delta-2 d$, so $\tilde{\Delta}$ is the usual finite difference Laplacian on $\mathbf{Z}^{d}$. Then $C=\left(-\tilde{\Delta}+m^{2}\right)^{-1}$ satisfies the desired hypotheses in any dimension if $m^{2}>0$. The same is true for $C=(-\tilde{\Delta})^{-1}$ if $d \geqq 3$. Notice that for $C=(-\tilde{\Delta})^{-1}$ and $d=3$ we have $\theta=1$.

Theorem 2.2 will be proven in Sect. 4. The fact that $V$ is a Wegner potential is a consequence of (2.3). To show $V$ is of type $\left(\alpha, \rho, 2^{k}\right)$, we will derive a multivariable version of Nelson's best possible hypercontractive estimate from which the result will follow.

Our second example concerns Gibbs potentials, i.e., potentials whose probability distribution is given by a Gibbs measure, which we are going to require to be completely analytical in the sense of Dobrushin and Shlosman [23, 24]. We will call such potentials completely analytical Gibbs potentials. 
Theorem 2.3. Let $V$ be a completely analytical Gibbs potential with the "a priori" single site spin distribution absolutely continuous with compact support and bounded Radon-Nikodym derivative. Then $V$ is a Wegner potential of type $(\alpha, K, K)$ for any $1<\alpha<K$. In particular, $H=-\Delta+V$ exhibits polynomial localization at high disorder or low energy, in the precise sense of Theorem 2.1.

The proof of Theorem 2.3 is given in Sect. 5. That $V$ is a Wegner potential follows from the DLR equations and our assumptions on the "a priori" spin distribution, since completely analytical Gibbs fields have finite range interactions. The proof that $V$ is of type $(\alpha, K, K)$ is due to Senya Shlosman [28].

Examples of completely analytical Gibbs fields are given by high-temperature Gibbs fields, or ferromagnetic Gibbs fields with a large magnetic field (arbitrary temperature) [23].

\section{The Multiscale Analysis}

We start by recalling a Simon-Lieb type inequality for Green's functions that follows from the resolvent identity $[18,6,7,21,9]$. Let

$$
\Lambda \subset \Omega \subset \mathbf{Z}^{d}, E \in \mathbf{R}, \varepsilon \neq 0, x \in \Lambda, y \in \Omega \backslash \Lambda .
$$

We have

$$
G_{\Omega}(E+i \varepsilon ; x, y)=\sum_{\left\langle u, u^{\prime}\right\rangle \in \partial(\Lambda, \Omega)} G_{\Lambda}(E+i \varepsilon ; x, u) G_{\Omega}\left(E+i \varepsilon ; u^{\prime}, y\right),
$$

where

$$
\partial(\Lambda, \Omega)=\left\{\left\langle u, u^{\prime}\right\rangle ; u \in \Lambda, u^{\prime} \in \Omega \backslash \Lambda,\left|u-u^{\prime}\right|=1\right\}
$$

We write

$$
\begin{aligned}
\partial \Lambda & =\partial\left(\Lambda, \mathbf{Z}^{d}\right), \\
\partial \Lambda^{+} & =\left\{u^{\prime} \in \mathbf{Z}^{d} \backslash \Lambda ;\left\langle u, u^{\prime}\right\rangle \in \partial \Lambda \text { for some } u \in \Lambda\right\}, \\
\partial \Lambda^{-} & =\left\{u \in \Lambda ;\left\langle u, u^{\prime}\right\rangle \in \partial \Lambda \text { for some } u^{\prime} \in \mathbf{Z}^{d} \backslash \Lambda\right\},
\end{aligned}
$$

and

$$
G_{\Lambda}(E+i \varepsilon ; x, \partial)=\sum_{\left\langle u, u^{\prime}\right\rangle \in \partial \Lambda}\left|G_{\Lambda}(E+i \varepsilon ; x, u)\right|
$$

Thus

$$
\left|G_{\Omega}(E+i \varepsilon ; x, y)\right| \leqq G_{\Lambda}(E+i \varepsilon ; x, \partial)\left|G_{\Omega}\left(E+i \varepsilon ; u^{\prime \prime}, y\right)\right|
$$

for some $u^{\prime \prime} \in \partial \Lambda^{+} \cap \Omega$.

Definition. Let $\eta>0, E \in \mathbf{R}, L>0$. A site $x \in \mathbf{Z}^{d}$ is $(\eta, E, L)$-regular (for a fixed $V$ ) if

$$
\tilde{G}_{\Lambda_{L}(x)}(E ; x, \partial) \equiv \sup _{\varepsilon \neq 0} \mid G_{\Lambda_{L}(x)}(E+i \varepsilon ; x, \partial) \leqq \frac{1}{L^{\eta}}
$$

A set $\Lambda \subset \mathbf{Z}^{d}$ is $(\eta, E, L)$ regular if every $x \in \Lambda$ is $(\eta, E, L)$-regular. 
Let $x \in \Omega \subset \mathbf{Z}^{d}$ with $\Lambda_{L}(x) \cup \partial \Lambda_{L}(x)^{+} \subset \Omega$. If $x$ is $(\eta, E, L)$-regular, it follows from (3.1) that for all $y \in \Omega \backslash \Lambda_{L}(x)$ and $\varepsilon \neq 0$,

$$
\left|G_{\Omega}(E+i \varepsilon ; x, y)\right| \leqq \frac{1}{L^{\eta}}\left|G_{\Omega}(E+i \varepsilon ; v, y)\right|
$$

for some $v \in \partial \Lambda_{L}(x)^{+}$.

Theorem 3.1. Let $V$ be a Wegner potential of type $(\alpha, \rho K)$ for some $1<\alpha<\rho$, $K \in\{2,3,4, \ldots\}$. Suppose

$$
\mathbf{P}\left\{0 \text { is }\left(\eta, E, L_{0}\right) \text {-regular }\right\} \geqq 1-\frac{1}{L_{0}^{r}}
$$

for some

$$
E \in \mathbf{R}, L_{0}>1, r>\frac{\alpha(d-1) K}{\rho-\alpha}, \eta(A-\alpha)>(\beta+d-1)(K-1+\alpha(A+K-1)),
$$

where $A>\alpha$ is an integer and $\beta>\alpha r+(A+K-1)(d-1)+d$.

Let $L_{k+1}=(A+K-1) L_{k}^{\alpha}, \quad k=0,1,2, \ldots$. Then, there exists $\bar{L}=\bar{L}(\alpha, d, K, r, A, \beta, \eta)>0$, such that if $L_{0}>\bar{L}$, we have

$$
\mathbf{P}\left\{0 \text { is }\left(\eta, E, L_{k}\right) \text {-regular }\right\} \geqq 1-\frac{1}{L_{k}^{r}}
$$

for all $k=0,1,2, \ldots$.

Theorem 3.2. Let $V$ be a Wegner potential, such that for some $E \in \mathbf{R}, \alpha>1, D \geqq 1$, $L_{0}>1, r>\alpha d, \eta>\alpha d$, we have

$$
\mathbf{P}\left\{0 \text { is }\left(\eta, E, L_{k}\right) \text {-regular }\right\} \geqq 1-\frac{1}{L_{k}^{r}}
$$

for all $k=0,1,2, \ldots$, where $L_{k+1}=D L_{k}^{\alpha}$. Let $0<\bar{\eta}<\frac{\eta-\alpha d}{\alpha}$. We have, with probability one,

$$
\sup _{\varepsilon \neq 0}|G(E+i \varepsilon ; 0, x)| \leqq \frac{C}{1+\|x\|^{\dot{\eta}}}
$$

for all $x \in \mathbf{Z}^{d}$ for some $C=C(V, \bar{\eta})<\infty$.

Theorems 3.1 and 3.2 prove Theorem 2.1. Condition (3.3) is satisfied at high disorder or low energy (see, for instance, the discussion in [9]). If in (3.4) we have $\bar{\eta}>d / 2$, we can apply the results of Simon and Wolff [20] to conclude pure point spectrum with probability one, and that the corresponding eigenfunctions have at least the rate of decay of the Green's function.

Proof of Theorem 3.1. Given $L>0$, let $R_{L}$ be the statement

$$
\mathbf{P}\{0 \text { is }(\eta, E, L) \text {-regular }\} \geqq 1-\frac{1}{L^{r}} .
$$

Theorem 3.1 follows from 
Lemma 3.3. Let $V, \alpha, K, \rho, r, \eta, A, \beta$ be as in Theorem 3.1. Suppose $R_{l}$ holds, and let $L=(A+K-1) l^{\alpha}$. Then $R_{L}$ holds if l is large enough.

Proof. Let $D=A+K-1$, so $L=D l^{\alpha}$. We set $\Lambda_{j}=\Lambda_{j l}(0), j=1, \ldots, D$. We also set $\Lambda=\Lambda_{D}=\Lambda_{L}(0)$. have

Let $y \in \partial \Lambda^{-}$. We will estimate $G_{\Lambda}(E+i \varepsilon ; 0, y)$ by applying (3.1) repeatedly. We

$$
\begin{aligned}
\left|G_{\Lambda}(E+i \varepsilon ; 0, y)\right| \leqq & G_{\Lambda_{l}(0)}(E+i \varepsilon ; 0, \partial)\left|G_{\Lambda}\left(E+i \varepsilon ; v_{0}, y\right)\right| \\
\leqq & G_{\Lambda_{l}(0)}(E+i \varepsilon ; 0, \partial) G_{\Lambda_{1}}\left(E+i \varepsilon ; v_{0}, \partial\right) \mid G_{\Lambda}\left(E+i \varepsilon ; u_{1}, y\right) \\
\leqq & G_{\Lambda_{l}(0)}\left(E+i \varepsilon ; v_{0}, \partial\right) G_{\Lambda_{1}}\left(E+i \varepsilon ; v_{0}, \partial\right) G_{\Lambda_{l}\left(u_{1}\right)}\left(E+i \varepsilon ; u_{1}, \partial\right) \\
& \cdot G_{\Lambda_{2}}\left(E+i \varepsilon ; v_{1}, \partial\right) G_{\Lambda_{l}\left(u_{2}\right)}\left(E+i \varepsilon ; u_{2}, \partial\right) \\
& \ldots G_{\Lambda_{D-1}}\left(E+i \varepsilon ; v_{D-1}, \partial\right) G_{\Lambda_{l}\left(u_{D-1}\right)}\left(E+i \varepsilon ; u_{D-1}, \partial\right) \\
& \cdot\left|G_{\Lambda}\left(E+i \varepsilon ; v_{D-1}, y\right)\right|
\end{aligned}
$$

for some $v_{0} \in \partial \Lambda_{l}(0)^{+}, u_{j} \in \partial \Lambda_{j}^{+}, v_{j} \in \partial \Lambda_{l}\left(u_{j}\right)^{+}, j=1, \ldots, D-1$.

We will call a cube $\Lambda_{t}(x)(E, \beta)$-non resonant $((E, \beta)-N R)$ if

$$
d\left(E, \sigma\left(H_{\Lambda_{t}(x)}\right)\right)>\frac{1}{t^{\beta}} .
$$

Notice also that $\left|\partial \Lambda_{t}(x)^{-}\right| \leqq\left|\partial \Lambda_{t}(x)^{+}\right| \leqq s t^{d-1}$ for some $s=s(d)<\infty$. Thus, if $\Lambda_{t}(x)$ is $(E, \beta)-N R$, we have

$$
G_{\Lambda_{t}(x)}(E+i \varepsilon ; u, \partial) \leqq s t^{\beta+d-1} .
$$

Let $\mathscr{E}$ be the event defined by

(i) All $\Lambda_{j}, j=1, \ldots, D$, and $\Lambda_{l}(x), x \in \Lambda$, are $(E, \beta)-N R$.

(ii) There exist $j_{1}<j_{2}<\cdots<j_{A} \in\{0,1, \ldots, D-1\}$, such that $\partial \Lambda_{j_{1}}^{+}, \ldots, \partial \Lambda_{j_{A}}^{+}$are $(\eta, E, l)$-regular, where $\partial \Lambda_{0}^{+}=\{0\}$.

Let us now assume that the event $\mathscr{E}$ holds. It follows from (3.5) and (3.6) that

$$
\begin{aligned}
G_{\Lambda}(E+i \varepsilon ; 0, \partial) & \leqq \frac{1}{l^{A \eta}}\left(s l^{\beta+d-1}\right)^{K-1}\left(s L^{\beta+d-1}\right)^{D} \\
& =s^{K-1+D} D^{D} l^{-[A \eta-(\beta+d-1)(K-1+\alpha D)]} \leqq \frac{1}{L^{\eta}}=\frac{1}{D^{\eta} l^{\alpha \eta}}
\end{aligned}
$$

for $l$ sufficiently large, since

$$
\eta(A-\alpha)>(\beta+d-1)(K-1+\alpha(A+K)) .
$$

Thus, to conclude the proof of the lemma we need only to show that

$$
\mathbf{P}(\mathscr{E}) \geqq 1-\frac{1}{L^{r}}
$$

Since $V$ is a Wegner potential, it follows from (2.1) that

$$
\mathbf{P}\left\{\Lambda_{t}(x) \text { is }(E, \beta)-N R\right\} \geqq 1-\frac{\sigma}{t^{\beta-d}}
$$


for some $\sigma>0$, depending only on $V$. Thus

$$
\mathbf{P}\left\{(\text { i) does not hold }\} \leqq \frac{D \sigma}{l^{\alpha(\beta-d)}}+\frac{(L+1)^{d} \sigma}{l^{\beta-d}} \leqq \frac{1}{2 L^{r}}\right.
$$

for $l$ sufficiently large, since $\alpha r<\beta-(\alpha+1) d$.

So it only remains to show that

$$
P\{\text { (ii) does not hold }\} \leqq \frac{1}{2 L^{r}} \text {. }
$$

We have

$$
\begin{aligned}
& \mathbf{P}\{\text { (ii) does not hold }\}=\mathbf{P}\left\{\text { there exist } u_{j_{i}} \in \partial \Lambda_{j_{i}}^{+} \text {not }(\eta, E, l)\right. \text {-regular } \\
& \left.i=1, \ldots, K, j_{1}<j_{2}<\cdots<j_{K} \in\{0,1, \ldots, D-1\}\right\} \\
& \leqq \sum_{j_{1}<j_{2}<\cdots j_{K} \in\{0, \ldots D-1\}} \sum_{u_{i} \in \partial \Lambda_{i}^{+}, i=1, \ldots, K} \mathbf{P}\left\{u_{1}, \ldots, u_{K} \text { not }(\eta, E, L) \text {-regular }\right\} .
\end{aligned}
$$

Since $\left\|u_{i^{\prime}}-u_{i}\right\| \geqq \frac{l^{\alpha}}{2}$ for $i \neq i^{\prime}$, we can use (2.2) for $l$ sufficiently large to conclude

$$
\mathbf{P}\{\text { (ii) does not hold }\} \leqq\left(\begin{array}{l}
D \\
K
\end{array}\right)\left(s L^{d-1}\right)^{K} \frac{C}{l^{\rho r}} \leqq \frac{1}{2 L^{r}}
$$

for $l$ sufficiently large since

$$
r>\frac{\alpha(d-1) K}{\rho-\alpha} .
$$

This completes the proof of Lemma 3.3 and hence of Theorem 3.1

Proof of Theorem 3.2. Let $0<\bar{\eta}<\frac{\eta-\alpha d}{\alpha}$, we choose $\beta>d$ by $\bar{\eta}=\frac{\eta-\alpha \beta}{\alpha}$.

Let us define the events $A_{k+1}=\left\{x\right.$ is $\left(\eta, E, L_{k}\right)$-regular for all $x \in \Lambda_{2 L_{k+1}}(0)$ and $\Lambda_{L_{k+1}}(0)$ is $\left.(E, \beta)-N R\right\}$.

By the hypotheses of Theorem 3.2, we have

$$
\mathbf{P}\left(A_{k+1}^{c}\right) \leqq \frac{\left(2 L_{k+1}+1\right)^{d}}{L_{k}^{r}}+\frac{\sigma}{L_{k+1}^{\beta-d}},
$$

so $\sum_{k=0}^{\infty} \mathbf{P}\left(A_{k+1}^{c}\right)<\infty$, since $r>\alpha d, \beta>d$. It follows from the Borel-Cantelli Lemma that, with probability one, we can find $\bar{k}=\bar{k}(V)<\infty$ such that $A_{k+1}$ holds for all $k \geqq \bar{k}$.

So let us fix a potential $V$ and $\bar{k}<\infty$ such that $A_{k+1}$ holds for $k \geqq k$. Given $x \in \mathbf{Z}^{d}$, we pick $\tilde{k}=\tilde{k}(x)$ in the following way:

(i) if $\|x\| \leqq \frac{1}{2} L_{\tilde{k}+1}$, pick $\tilde{k}=\bar{k}$.

(ii) if $\|x\|>\frac{1}{2} L_{\bar{k}+1}$, pick $\tilde{k}$ such that $\frac{1}{2} L_{\bar{k}}<\|x\| \leqq \frac{1}{2} L_{\bar{k}+1}$.

We let $G_{k}(E+i \varepsilon)=G_{\Lambda_{L_{k}(0)}}(E+i \varepsilon)$, and let $\Gamma_{k}$ be the operator defined by

$$
\Gamma_{k}\left(u, u^{\prime}\right)=\left\{\begin{array}{ll}
1 & \text { if either }\left\langle u, u^{\prime}\right\rangle \text { or }\left\langle u^{\prime}, u\right\rangle \in \partial \Lambda_{L_{k}}(0) \\
0 & \text { otherwise }
\end{array}\right. \text {. }
$$


It follows from the resolvent identity, applied repeatedly, that (we omit $E+i \varepsilon$ ) $G=G_{\tilde{k}+1}+G_{\tilde{k}+1} \Gamma_{\tilde{k}+1} G_{\tilde{k}+2}+\cdots+G_{\tilde{k}+1} \Gamma_{\tilde{k}+1} G_{\tilde{k}+2} \cdots \Gamma_{\tilde{k}+1+l} G_{\tilde{k}+2+l}+\cdots$. If $\tilde{k}=\bar{k}$, we just take

$$
\left|G_{\tilde{k}+1}(E+i \varepsilon ; 0, x)\right| \leqq L_{\bar{k}+1}^{\beta} .
$$

If $\tilde{k}>\bar{k}$, we use (3.2) $m$-times where

$$
m=\left[\frac{\|x\|}{\left[\frac{L_{\tilde{k}}}{2}\right]+1}\right]
$$

(here $[t]$ means the largest integer $\leqq t$ ), to obtain

$$
\left|G_{\tilde{k}+1}(E+i \varepsilon ; 0, x)\right| \leqq L_{\tilde{k}}^{-\eta m} L_{\tilde{k}+1}^{\beta} \leqq D^{\beta} L_{\tilde{k}}^{-(\eta-\beta \alpha)} \leqq D^{\beta}\left(\frac{D}{2}\right)^{(\eta-\beta \alpha) / \alpha} \frac{1}{\|x\|^{(\eta-\beta \alpha) / \alpha}},
$$

since $m \geqq 1,\|x\| \leqq \frac{1}{2} L_{\bar{k}+1}$.

Similarly

where

$$
\left|G_{\tilde{k}+1} \Gamma_{\tilde{k}+1} \ldots \Gamma_{\tilde{k}+1+l} G_{\tilde{k}+2+l}(0, x)\right| \leqq \frac{1}{L_{\tilde{k}+1}^{\eta}} \frac{1}{L_{\bar{k}+1}^{\eta \eta_{\tilde{k}+1}}} \cdots \frac{1}{L_{\tilde{k}+l}^{\eta \eta_{\tilde{k}+l}}} L_{\tilde{k}+2+l}^{\beta},
$$

$$
\gamma_{k}=\left[\frac{\left[\frac{L_{k+1}}{2}\right]-\left[\frac{L_{k}}{2}\right]-1}{\left[\frac{L_{k}}{2}\right]+1}\right],
$$

and thus

$$
\leqq \frac{C}{L_{\tilde{k}+1}^{(n-\alpha \beta)(l+1)}} \leqq \frac{C^{\prime}}{\|x\|^{((\eta-\alpha \beta) / \alpha(l)(l+1)}}
$$

for some constants $C, C^{\prime \prime}$ independent of $x$.

Thus, we have

$$
|G(E+i \varepsilon ; \partial, x)| \leqq L_{\tilde{k}+1}^{\beta}+\frac{C^{\prime \prime}}{\|x\|^{(\eta-\alpha \beta) / \alpha}}
$$

if $\|x\| \leqq \frac{L_{\tilde{k}+1}}{2}$, some constant $C^{\prime \prime}<\infty$ and

otherwise.

$$
|G(E+i \varepsilon ; 0, x)| \leqq \frac{C^{\prime \prime}}{\|x\|^{(\eta-\alpha \beta) / \alpha}}
$$

Recall $\bar{\eta}=\frac{\eta-\alpha \beta}{\alpha}$, it follows from (3.7) and (3.8) that

$$
|G(E+i \varepsilon ; 0, x)| \leqq \frac{C}{1+\|x\|^{\tilde{\eta}}}
$$

for all $x \in \mathbf{Z}^{d}$ for some constant $C<\infty$. 
This proves Theorem 3.2.

\section{Gaussian Potentials}

The key ingredient in the proof of Theorem 2.2 is Nelson's best possible hypercontractive estimate [30-35]. It can be reformulated as follows [35]:

Let $X, Y$ be jointly Gaussian random variables, both with zero mean. Then, for any measurable functions $f$ and $g$ of a real variable, we have

$$
|\mathbf{E}(f(X) g(Y))| \leqq\|f(X)\|_{p}\|g(Y)\|_{q}
$$

if

$$
(p-1)(q-1) \geqq \frac{\mathbf{E}(X Y)^{2}}{\mathbf{E}\left(X^{2}\right) \mathbf{E}\left(Y^{2}\right)}
$$

If particular, we can take

$$
p=q=1+\frac{|\mathbf{E}(X Y)|}{\left(\mathbf{E}\left(X^{2}\right) \mathbf{E}\left(Y^{2}\right)\right)^{1 / 2}} .
$$

We will need a multivariable version of this result.

Lemma. Let $X_{1}, X_{2}, \ldots, X_{n}, Y_{1}, Y_{2}, \ldots, Y_{n}$ be jointly Gaussian random variables with mean zero. Let

$$
\mathbf{X}=\left(X_{1}, \ldots, X_{n}\right), \mathbf{Y}=\left(Y_{1}, \ldots, Y_{n}\right), P_{1}=\mathbf{E}\left(\mathbf{X}^{t} \mathbf{X}\right), P_{2}=\mathbf{E}\left(\mathbf{Y}^{t} \mathbf{Y}\right), Q=\mathbf{E}\left(\mathbf{X}^{t} \mathbf{Y}\right) .
$$

Then, for any measureable functions $f, g$ on $\mathbf{R}^{n}$, we have

$$
\mid \mathbf{E}\left(f(\mathbf{X}) g(\mathbf{Y}) \mid \leqq\|f(\mathbf{X})\|_{p}\|g(\mathbf{Y})\|_{q}\right.
$$

if

$$
(p-1)(q-1) \geqq\left\|P_{1}^{-1 / 2} Q P_{2}^{-1 / 2}\right\|^{2},
$$

where we used the operator norm for matrices. In particular, we can take

$$
p=q=1+\left\|P_{1}^{-1 / 2} Q P_{2}^{-1 / 2}\right\| .
$$

Proof. By a limiting procedure we can take the covariance matrix

$$
C=\left(\begin{array}{ll}
P_{1} & Q \\
Q^{t} & P_{2}
\end{array}\right)
$$

to be strictly positive definite. In this case we can also take

$$
C=\left(\begin{array}{cc}
I & Q \\
Q^{t} & I
\end{array}\right)
$$

without loss of generality.

Since $C>0,\|Q\|<1$, and we have

$$
C^{-1}=\left[\begin{array}{cc}
\left(I-Q Q^{t}\right)^{-1} & -\left(I-Q Q^{t}\right)^{-1} Q \\
-\left(I-Q^{t} Q\right)^{-1} Q^{t} & \left(I-Q^{t} Q\right)^{-1}
\end{array}\right] .
$$

Let us consider the operator on $\mathscr{K}=L_{2}\left(\mathbf{R}^{n},(2 \pi)^{-n / 2} e^{(-1 / 2)\left(|x|^{2}\right)} d \mathbf{x}\right)$ given by the 
multivariable Mehler's formula [34; (I.34)]:

$$
(\Gamma(Q) f)(\mathbf{x})=(2 \pi)^{-n / 2}\left[\operatorname{det}\left(1-Q^{t} Q\right)\right]^{1 / 2} \int e^{(-1 / 2)\left\langle\left(Q^{t} \mathbf{x}-\mathbf{y}\right),\left(1-Q^{t} Q\right)^{-1}\left(Q^{t} \mathbf{x}-\mathbf{y}\right)\right\rangle} f(\mathbf{y}) d \mathbf{y}
$$

It is not hard to check that

$$
\mathbf{E}(f(X) g(Y))=\langle f, \Gamma(Q) g\rangle_{\mathscr{X}} .
$$

The lemma now follows from Nelson's best possible hypercontractive estimate (see [34; Theorem I.17]).

We are now ready to prove Theorem 2.2. We first show that it follows from (2.3) that $V$ is a Wegner potential. Let $0 \in J \subset \mathbf{Z}^{d}$ finite, an explicit computation shows that the conditional probability distribution of $V(0)$ given $V_{J \backslash\{0\}}=$ $\{V(x) ; x \in J \backslash\{0\}\}$ is absolutely continuous with density

$$
\left.f_{J}\left(V(0) \mid V_{J \backslash\{0\}}\right)=\sqrt{\frac{a_{J}}{2 \pi}} e^{(-1 / 2) a_{J}\left(V(0)+\left(1 / a_{J}\right)\right.} \sum_{x \in \backslash \backslash 0\}} C_{J}^{-1}(0, x) V(x)\right)^{2},
$$

where $a_{J}=C_{J}^{-1}(0,0)$. By $(2.3)$ we have

$$
\left\|f_{J}\left(V(0) \mid V_{J \backslash\{0\}}\right)\right\|_{\infty} \leqq \sqrt{\frac{a}{2 \pi}}<\infty .
$$

We can conclude that the conditional probability distribution of $V(0)$ given $V(0)^{\perp}$ is absolutely continuous with a density bounded by $\sqrt{\frac{a}{2 \pi}}$.

Now let $0 \leqq \tau \leqq d$ be such that $\left\|C_{J}^{-1}\right\| \leqq c_{1}|J|^{\tau / d}$ for all $J \subset \mathbf{Z}^{d}$ finite, some $c_{1}<\infty$. This can always be done since

$$
\left\|C_{J}^{-1}\right\| \leqq \operatorname{Tr} C_{J}^{-1} \leqq a|J|
$$

by (2.3).

So let us pick $\alpha, \rho$, and an integer $k$ such that $\max \left\{1, \frac{d+r}{\theta}\right\}<\alpha<\rho<2^{k}$. It will follow from the following lemma that $V$ is of type $\left(\alpha, \rho, 2^{k}\right)$.

Lemma 4.2. Let $L>2 l>0, x_{i} \in \mathbf{Z}^{d}, i=1, \ldots, 2^{k}$, with $\left\|x_{i}-x_{j}\right\| \geqq L$ for $i \neq j$, and let $A_{i}$ be events on $\Lambda_{l}\left(x_{i}\right), i=1, \ldots, 2^{k}$. Then

$$
\mathbf{P}\left(\bigcap_{i=1}^{2^{k}} A_{i}\right) \leqq \prod_{i=1}^{2^{k}} \mathbf{P}\left(A_{i}\right)^{(1 / p)^{k}}
$$

with

$$
p \geqq 1+b c_{1} 4^{k-1} \frac{(l+1)^{r+d}}{1+(L-l)^{\theta}}
$$

In particular, if $A$ is an event on $\Lambda_{l}(0)$, we have

$$
\mathbf{P}\left(\bigcap_{i=1}^{2^{k}} A\left(x_{i}\right)\right) \leqq \mathbf{P}(A)^{(2 / p)^{k}}
$$


Proof. Let $I_{1}=\left\{1, \ldots, 2^{k-1}\right\}, I_{2}=\left\{2^{k-1}+1, \ldots, 2^{k}\right\}$. We apply Lemma 4.1 with

$$
\begin{aligned}
& \mathbf{X}=\left\{V(y) ; y \in \Lambda_{l}\left(x_{i}\right), i \in I_{1}\right\}, \\
& \mathbf{Y}=\left\{V(y) ; y \in \Lambda_{l}\left(x_{i}\right), i \in I_{2}\right\}
\end{aligned}
$$

Then

$$
\mathbf{P}\left(\bigcap_{i=1}^{2^{k}} A_{i}\right) \leqq \mathbf{P}\left(\bigcap_{i \in I_{1}} A_{i}\right)^{1 / p} \mathbf{P}\left(\bigcap_{i \in I_{2}} A_{i}\right)^{1 / p},
$$

where

$$
p \geqq 1+\left\|P_{1}^{-1 / 2} Q P_{2}^{-1 / 2}\right\|,
$$

$P_{1}, P_{2}, Q$ being defined as in Lemma 4.1. But

$$
\left\|P_{j}^{-1}\right\| \leqq c_{1}\left(2^{k-1}(l+1)^{d}\right)^{\tau / d}=c_{1} 2^{(k-1)(\tau / d)}(l+1)^{\tau},
$$

and we can estimate $\|Q\|$ by its Hilbert-Schmidt norm as follows using (2.4):

$$
\|Q\| \leqq 2^{(k-1)}(l+1)^{d} \frac{b}{1+(L-l)^{\theta}} .
$$

Thus

$$
p \geqq 1+b c_{1} 4^{k-1} \frac{(l+1)^{d+r}}{1+(L-l)^{\theta}} .
$$

Repeating the above procedure for a total of $k$-times, which we can do using always $p$ given by (4.1), we get the lemma.

This finishes the proof of Theorem 2.4.

\section{Gibbs Potentials}

In this section we present a proof of Theorem 2.3, due to S. Shlosman [28].

So let $V$ be as in Theorem 2.3. Since the DLR equations and our assumptions immediately imply that $V$ is a Wegner potential, we have only to prove that $V$ is of type $(\alpha, K, K)$ for any $1<\alpha<K$.

Dobrushin and Shlosman [23, 24] gave several equivalent conditions that characterize completely analytical Gibbs measures. We will show that their condition IIId implies that $V$ is of type $(\alpha, K, K)$ for any $1<\alpha<K$.

First, some notation. We will denote by $r$ the range of the interaction (by definition completely analytical interactions have finite range, i.e., $r<\infty$ ). Given $\Lambda \subset \mathbf{Z}^{d}$, we define

$$
\begin{aligned}
\partial \Lambda & =\left\{y \in \mathbf{Z}^{d} \backslash \Lambda ; \operatorname{dist}(y, \Lambda) \leqq r\right\}, \\
V_{\Lambda} & =\{V(x) ; x \in \Lambda\},
\end{aligned}
$$

$\mathbf{P}_{\Lambda}\left(\cdot \mid V_{\Lambda^{c}}\right)=$ conditional probability distribution of $V_{\Lambda}$ given $V_{\Lambda^{c}}$, where $\Lambda^{c}=\mathbf{Z}^{d} \backslash \Lambda$.

We can now state Dobrushin and Shlosman's condition IIId:

There exist $C<\infty, \gamma>0$, such that for all $\Lambda \subset \Omega \subset \mathbf{Z}^{d}$ finite, any $y \in \partial \Omega$, and 
any choices $V_{1}, V_{2}$ of $V_{\Omega^{c}}$, such that $V_{1}(x)=V_{2}(x)$ for $x \in \Omega^{c} \backslash\{y\}$, we have

$$
\left|\frac{\mathbf{P}_{\Omega}\left(A \mid V_{1}\right)}{\mathbf{P}_{\Omega}\left(A \mid V_{2}\right)}-1\right| \leqq C e^{-\gamma \operatorname{dist}(y, A)}
$$

for any event in $A$ in $\Lambda$.

Lemma 5.1. For all $\Lambda \subset \Omega \subset \mathbf{Z}^{d}$ finite, any choices $V_{1}, V_{2}$ of $V_{\Omega^{c}}$ we have

$$
\left|\frac{\mathbf{P}_{\Omega}\left(A \mid V_{1}\right)}{\mathbf{P}_{\Omega}\left(A \mid V_{2}\right)}-1\right| \leqq\left(1+C e^{-\gamma \operatorname{dist}(\Lambda, \partial \Omega)}\right)^{|\partial \Omega|}-1 \text {. }
$$

Proof. Without loss of generality we can always assume $V_{1}(x)=V_{2}(x)$ for $x \in \Omega^{c} \backslash \partial \Omega$. For such $V_{1}, V_{2}$ we can always find values $W_{0}, W_{1}, \ldots, W_{k}$ of $V_{\Omega^{c}}$, such that $W_{0}=V_{1}, W_{k}=V_{2}, k \leqq|\partial \Omega|$, and for each $i=1, \ldots, k$ we can find $y_{i} \in \partial \Omega$ such that $W_{i-1}(x)=W_{i}(x)$ for $x \neq y_{i}$. We have

$$
\frac{\mathbf{P}_{\Omega}\left(A \mid V_{1}\right)}{\mathbf{P}_{\Omega}\left(A \mid V_{2}\right)}=\prod_{i=1}^{k} \frac{\mathbf{P}_{\Omega}\left(A \mid W_{i-1}\right)}{\mathbf{P}_{\Omega}\left(A \mid W_{i}\right)} .
$$

Equation (5.1) applies to each factor in the right-hand-side so we can conclude that for each $i=1, \ldots, k$, we have

$$
1-K e^{-\gamma \operatorname{dist}(\Lambda, \partial \Omega)} \leqq \frac{\mathbf{P}_{\Omega}\left(A \mid W_{i-1}\right)}{\mathbf{P}_{\Omega}\left(A \mid W_{i}\right)} \leqq 1+K e^{-\gamma \operatorname{dist}(\Lambda, \partial \Omega)},
$$

and hence

$$
-\left(1-\left(1-K e^{\left.-\Gamma \operatorname{dist}\left(\Lambda, \partial_{\Lambda}\right)\right)^{|\partial \Omega|}}\right) \leqq \frac{\mathbf{P}_{\Omega}\left(A \mid V_{1}\right)}{\mathbf{P}_{\Omega}\left(A \mid V_{2}\right)}-1 \leqq\left(1+K e^{-\gamma \operatorname{dist}\left(\Lambda, \partial_{\Lambda}\right)}\right)^{|\partial \Omega|}-1,\right.
$$

so (5.2) follows.

If $\operatorname{dist}(\Lambda, \partial \Omega)$ is sufficiently large, we have

$$
|\partial \Omega| \leqq C_{1}[\operatorname{dist}(\Lambda, \partial \Omega)]^{d-1}
$$

for some fixed constant $C_{1}<\infty$. In this case, it follows from (5.2) that we can find $C^{\prime}<\infty, \gamma^{\prime}>0$, such that

$$
\left|\frac{\mathbf{P}_{\Omega}\left(A \mid V_{1}\right)}{\mathbf{P}_{\Omega}\left(A \mid V_{2}\right)}-1\right| \leqq C^{\prime} e^{-\gamma^{\prime} \operatorname{dist}(\Lambda, \partial \Omega)}
$$

Now, let $B$ be an event on $\Omega^{c}$. It follows from (5.5) that

$$
\left|\frac{\mathbf{P}_{\Omega}(A \mid B)}{\mathbf{P}(A)}-1\right| \leqq C^{\prime} e^{-\gamma \operatorname{dist}(\Lambda, \partial \Omega)} .
$$

We are now ready to show $V$ is of type $(\alpha, K, K)$ for $1<\alpha<K$. For given events $A_{1}, A_{2}, \ldots, A_{K}$, we always have

$$
\mathbf{P}\left(\bigcap_{i=1}^{K} A_{i}\right)=\mathbf{P}\left(A_{1}\right) \mathbf{P}\left(A_{2} \mid A_{1}\right) \mathbf{P}\left(A_{3} \mid A_{2} \cap A_{1}\right) \ldots \mathbf{P}\left(A_{K} \mid \bigcap_{i=1}^{K-1} A_{i}\right) .
$$

So let $1<l<L, x_{1}, \ldots, x_{K} \in \mathbf{Z}^{d}$, with $\left\|x_{i}-x_{j}\right\|>L$ for all $i \neq j$. Assume $L-l$ is sufficiently large. Then, if $A_{i}$ is an event on $\Lambda_{l}\left(x_{i}\right), i=1, \ldots, K$, we have from (5.4) 
that, for each $i=1, \ldots, K$,

$$
\frac{\mathbf{P}\left(A_{i} \mid \bigcap_{j=1}^{i-1} A_{j}\right)}{\mathbf{P}\left(A_{i}\right)}-1 \leqq C^{\prime} e^{-\gamma^{\prime}(L-l)}
$$

so

$$
\mathbf{P}\left(A_{i} \mid \bigcap_{j=1}^{i-1} A_{j}\right) \leqq \mathbf{P}\left(A_{i}\right)\left(1+C^{\prime} e^{-\gamma^{\prime}(L-l)}\right) .
$$

Thus, it follows from (5.5) that

$$
\mathbf{P}\left(\bigcap_{i=1}^{K} A_{i}\right) \leqq \prod_{i=1}^{K} \mathbf{P}\left(A_{i}\right)\left(1+C^{\prime} e^{-\gamma^{\prime}(L-l)}\right)^{K} \leqq 2^{K} \prod_{i=1}^{K} \mathbf{P}\left(A_{i}\right)
$$

This proves Theorem 2.3.

\section{References}

1. Anderson, P.: Absence of diffusion in certain random lattices. Phys. Rev. 109, 1492 (1958)

2. Pastur, L. A.: Spectra of random self adjoint operators. Russ. Math. Surv. 28, 1 (1973)

3. Kunz, H., Souillard, B.: Sur le spectre des operateurs aux differences finies aleatoires. Commun. Math. Phys. 78, 201-246 (1980)

4. Carmona, R., Lacroix, J.: Spectral theory of random Schrödinger operators. Boston, MA: Birkhauser 1990

5. Gol'dsheid, Ya., Molchanov, S., Pastur, L.: Pure point spectrum of stochastic one dimensional Schrödinger operators. Funct. Anal. Appl. 11, 1 (1977)

6. Frölich, J., Martinelli, F., Scoppola, E., Spencer, T.: Constructive proof of localization in the Anderson tight binding model. Commun. Math. Phys. 101, 21-46 (1985)

7. Martinelli, F., Scoppola, E.: Introduction to the mathematical theory of Anderson localization. Riv. Nuovo Cim. 10, N.10 (1987)

8. Carmona, R., Klein, A., Martinelli, F.: Anderson localization for Bernoulli and other singular potentials. Commun. Math. Phys. 108, 41-66 (1987)

9. von Dreifus, H., Klein, A.: A new proof of localization in the Anderson tight binding model. Commun. Math. Phys. 124, 285-299 (1989)

10. Lacroix, J.: Localisation pour l'opérateur de Schrödinger aléatoire dans un ruban. Ann. Inst. H. Poincaré ser A40, 97-116 (1984)

11. Klein, A., Lacroix, J., Speis, A.: Localization for the Anderson model on a strip with singular potentials. J. Funct. Anal. 94, 135-155 (1990)

12. Simon, B.: Localization in general one dimensional random systems, I. Jacobi matrices. Commun. Math. Phys. 102, 327-336 (1985)

13. Delyon, F., Levy, Y., Souillard, B.: Anderson localization for one and quasi one-dimensional systems. J. Stat. Phys. 41, 375 (1985)

14. Pastur, L. A.: Random and almost periodic operators: New examples of spectral behaviour. In: IXth International Congress on Mathematical Physics, Simon, B., Truman, A., Davies, I. M.,(eds.). Bristol, UK: Adam Hilger 1989

15. Sinai, Ya. G.: Anderson localization for one-dimensional difference Schrödinger operator with quasi-periodic potential. J. Stat. Phys. 46, 861-909 (1987)

16. Chulaevski, V., Sinai, Ya. G.: Anderson localization for the $1-D$ discrete Schrödinger operator with two-frequency potential. Commun. Math. Phys. 125, 91-112 (1989)

17. Fröhlich, J., Spencer, T., Wittwer, P.: Localization for a class of one-dimensional quasiperiodic Schrödinger operators Commun. Math. Phys. 132, 5-25 (1990)

18. Fröhlich, J., Spencer, T.: Absence of diffusion in the Anderson tight binding model for large disorder or low energy. Commun. Math. Phys. 88, 151-184 (1983) 
19. Delyon, F., Levy, Y., Souillard, B.: Anderson localization for multidimensional systems at large disorder or low energy. Commun. Math. Phys. 100, 463-470 (1985)

20. Simon, B., Wolff, T.: Singular continuum spectrum under rank one perturbations and localization for random Hamiltonians. Commun. Pure. Appl. Math. 39, 75-90 (1986)

21. von Dreifus, H.: On the effects of randomness in ferromagnetic models and Schrödinger operators. Ph.D. thesis, New York University (1987)

22. Spencer, T.: Localization for random and quasi-periodic potentials. J. Stat. Phys. 51, 1009 (1988)

23. Dobrushin, R. L., Shlosman, S. B.: Completely analytical Gibbs fields. In: Statistical Physics and Dynamical Systems, Fritz, J., Jaffe, A., Szasz, D., (eds.), pp. 371-403. Boston, MA: Birkhauser 1985

24. Dobrushin, R. L, Shlosman, S. B.: Completely analytical interactions: Constructive description. J. Stat. Phys. 46, 983-1014 (1987)

25. Wegner, F.: Bounds on the density of states in disordered systems. Z. Phys. B44, 9-15 (1981)

26. Grenander, V., Szego, G.: Toeplitz forms and their applications. New York, NY: Chelsea 1958

27. Grenander, V., Rosenblatt, M.: Statistical analysis of stationary time series. New York, NY: Chelsea 1957

28. Shlosman, S. B: Private communication

29. Nelson, E.: The free Markoff field. J. Funct. Anal. 12, 211-227 (1973)

30. Nelson, E.: Probability theory and Euclidean field theory. In: Constructive quantum field theory Velo, G., Wightman, A. (eds.). Berlin, Heidelberg, New York: Springer 1973

31. Gross, L.: Logarithmic Sobolev inequalities Am. J. Math. 97, 1061-1083 (1975)

32. Beckner, W.: Inequalities in Fourier analysis. Ann. Math. 102, 159-182 (1975)

33. Brascamp, H. J., Lieb, E. H.: Best constants in Young's inequalities, its converse, and its generalization to more than three functions. Adv. Math. 20, 151-173 (1976)

34. Simon, B.: The $P(\varnothing)_{2}$ Euclidean (Quantum) field theory. Princeton, NJ: Princeton University Press, 1974

35. Klein, A., Landau, L. J., Shucker, D. S.: Decoupling inequalities for stationary Gaussian processes. Ann. Prob. 10, 702-708 (1982)

Communicated by T. Spencer 
